\title{
Process Sequence Planning Modeling Based on Polychromatic Sets
}

\author{
Lei Qi ${ }^{1}$, Li Xian-wang ${ }^{2}$, Guo Wei-fei ${ }^{1}$ and Li Xiao-xin ${ }^{1}$ \\ ${ }^{1}$ State Key Laboratory of Mechanical Transmission, Chongqing University, \\ Chongqing, China \\ ${ }^{2}$ Guangxi University, Nanning, Guangxi, China \\ leiqi@cqu.edu.cn
}

\begin{abstract}
In view of the existing sequence planning problem, this paper presents a process scheduling model based on polychromatic sets theory. To attain the similarity and individuality of constraints of parts, the process route decision was divided into stages. A feature machining chain and process feature ranking model were built beginning with the factors that influence the processing stage and technology feature. The adoption of polychromatic sets theory simplifies the evaluation of process constraints; thus, knowledge can be expressed easily. This method not only ensures the versatility of the system, but also satisfies the customer-oriented process resource.
\end{abstract}

Keywords: sequence planning, polychromatic sets theory, constraint matrix

\section{Introduction}

Process planning is the art of optimizing the configuration of process resource and reasonably arranging the process. Based on the digitization demand for manufacturing process planning, many process planning systems have emerged on the basis of domestic and international research. Research methods according to the process of reasoning and decision logic are mainly divided into three types. The first type includes similarity-based methods, such as CBR investigated by Xiao Weiyue[1], and other reasoning and derivation methods based on similarity. These methods are suitable for a series of products; they require a precise instance library, appropriate search methods, and technology personnel with experience and knowledge. The second type includes expert systems. Zhang xiaodong[2] investigated expert systems, decision tables, the decision tree method, and so on. However, these methods are applicable only to simple parts reasoning. Liu Xiaoyang[3] proposed a reasoning mechanism based on the constraint matrix, which is mainly based on characteristics (working steps). Hu Yujin and Zhang Zhengyi[4] proposed a step-step matrix. The first type of method experiences difficulty expressing the mutual restraint relationship between two features; owing to the combined effect of the processing stages and characteristics, the second type of method is complex to determine the process and heavy workload. For the third type, Huang Weijun[5], ZhengYongqian[6], and Zhang Guanwei[7] investigated process planning based on the genetic algorithm. Alluru Gopala Krishna K[8] and Liu xiaojun[9] studied process planning based on the ant colony algorithm. These methods are still in the theoretical exploration stage. These three types take the optimization decision of the process resource and process sequencing decision together and thus make the system versatility poor.

To address the flaws of the above methods, this paper presents a process scheduling planning method based on polychromatic sets theory. The innovative points are as follows. First, the process route decision problem is divided into stages. The first stage only considers the common process constraints of parts and does not consider the constraints of the process resources and personality of parts. The second stage considers the constraints of the process resources and personality of parts. These two stages not 
only ensure the versatility of the system, but also make the process resources meet the customer's requirements. Second, in the process of establishing the rule of knowledge, a construct machining chain automatically generated model is featured. Third, part process characteristics are sorted in this part. The machining process is divided into three stages, namely, roughing, semi finishing, and finishing, According to the processing stages of the entire process, a decision is made in each stage for the process sequence of process features to simplify the process of knowledge expression and constrained judgment.

\section{Basic Concept of Polychromatic Sets Theory}

\subsection{Polychromatic Sets}

As a new type of mathematical modeling tool of system theory and information processing, the core idea of polychromatic sets theory is to simulate different objects (product, process design, process and production systems, etc.) through the same mathematical model, depict the hierarchy and complex relationships between elements, organize information in the collection and logic layers, and determine the size of the number in the number layer [10].

In polychromatic sets, a polychromatic set itself and its constituent elements are coated with different colors according to their different natures. The coloring for the overall nature of a polychromatic set is called unified color, and the coloring for elements is called personal color [10].

In polychromatic sets, the Boolean matrix $[A \times F(A)] \square$ between personal and unified coloring. is utilized to describe the relationship between personal and unified coloring.

\subsection{Concept of Polychromatic Sets Contour Comprising Matrix}

The advantage of polychromatic sets over traditional collection is that the former can express more information about the overall object and the component elements as well as the relationship between elements and overall objects or other elements through a different color. Polychromatic sets theory puts forward the concept of contour; it is an explanation for several concepts, including objects and attributes, nature, symbols, and parameters of the component elements. The same object can be described by its contour from the collection, logic, and number layers.

In the collection level, the contour matrix that indicates the basic component of object $\mathrm{A}$ is [11]

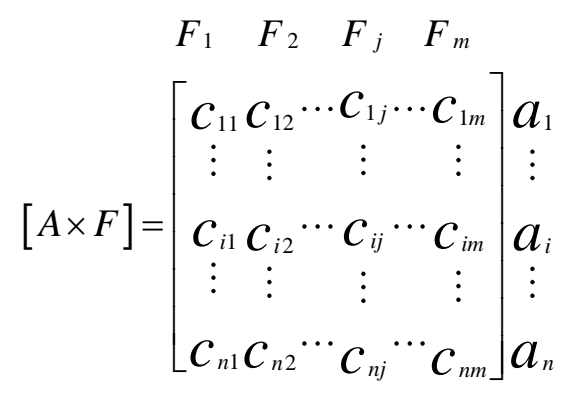

In the formula, the ingredients $c_{i j}=1$ determine the composition of $F\left(a_{i}\right)$. 


\section{Process Scheduling Model based on Polychromatic Sets Theory}

\subsection{Features: Working Step Mapping Rules}

The process features of the machined surface of a specific part are denoted as

$$
A=\left\{a_{1}, a_{2}, \cdots a_{i}, \cdots a_{n}\right\}
$$

The classification of features should be very clear and thinning because the same features have different processing methods under different circumstances, such as outer and inner circle surface.

All processing methods that are available to process the feature are denoted as

$$
F=\left\{F_{1}, F_{2}, \cdots F_{j}, \cdots F_{m}\right\}
$$

Therefore, the Boolean matrix of part features and the machining process step is expressed as Formula (1).

In the formula, ${ }^{c}{ }=1$ means the machining process characteristics can employ this method;

$c_{i j}=0$ indicates that the machining process characteristics cannot use this method.

Table 2.1 provides an example of an output shaft. It shows all available process means for each feature.

\begin{tabular}{|c|c|c|c|c|c|c|c|c|c|c|c|c|c|}
\hline & Turning & $\begin{array}{c}\text { Semi-fine } \\
\text { turning }\end{array}$ & $\begin{array}{l}\text { line } \\
\text { turnin } \\
g\end{array}$ & Bearizing & Reaming & Drilling & Milling & Boring & $\begin{array}{c}\text { Semi-fine } \\
\text { boring }\end{array}$ & $\begin{array}{l}\text { Fine } \\
\text { borin } \\
\mathrm{g}\end{array}$ & Grinding & $\begin{array}{l}\text { Accurate } \\
\text { grinding }\end{array}$ & Abrading \\
\hline Plane & $\bullet$ & - & • & & & & & & & & $\bullet$ & & \\
\hline Outer circle & $\bullet$ & - & - & & & & & & & & - & - & \\
\hline Hole & & & & $\bullet$ & - & - & & - & $\bullet$ & - & & $\bullet$ & $\bullet$ \\
\hline Key slot & & & & & & & $\bullet$ & & & & & & \\
\hline Chamfer & $\bullet$ & - & $\bullet$ & & & & & & & & & & \\
\hline
\end{tabular}

Table 2.1 Process Features-working Step Contour Boolean Matrix

\subsection{Process Step Chain Generation of Features of the Parts}

When formulating the process route, several methods can achieve the same aim, as shown in Table 2.1; however, this condition will cause repetition. Therefore, all the processing methods of the same feature were classified according to accuracy class constraints in this study by designing an algorithm to identify the number of processing chain of the same characteristic. This method can reduce the complexity of the manual input while laying the foundation for the subsequent steps and characteristics.

In a traditional polychromatic graph, each node represents a method. According to the relational model mapping rules proposed in Reference [1], in the extended polychromatic graph, we assumed that each node represents a machining precision level of a machining feature and is expressed as $u=\left\{u_{1}, u_{2}, \cdots u_{i}, \cdots u_{n}\right\}$.

Its start node $u_{i s}$ and end node ${ }^{u_{i e}}$ are the lower and upper limits of the limit range of machining accuracy level, respectively. The connection between two adjacent nodes 
represents the method utilized to accomplish processing at this precision level. The precision constraint model is structured based on the same technology features. Set of edges $\mathrm{C}$ can be obtained by calculating the Cartesian product of a set of nodes $U$.

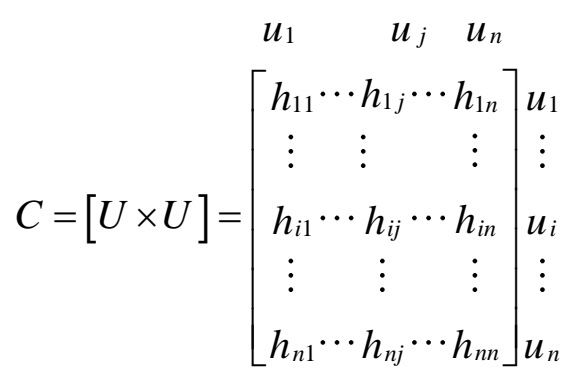

In the formula, $h_{i j}=1$ indicates that an edge exists between nodes u i and uj; all nodes similar to this form a set of edges C. Uniform coloring set of

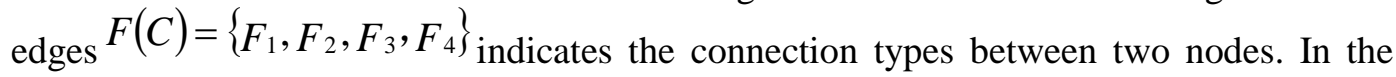
planning process, the connection between nodes is divided into three types: sequential connection, select connection ("or division" and "or close"), and blank connection. Sequential connection denotes that only one means exists to achieve the accuracy requirements (expressed by color $F_{1}$ ). Select connection indicates that a variety of means exist to achieve this accuracy. In select connection, the side relationship between the output node and select node is defined as "or division" and represented by color $F_{2}$. The side relationship between the select node and input node is defined as "or close" and marked by color $F_{3}$. Blank connection means no available process means exists; it is denoted by color $F_{4}$. The three basic connection model structures, the PS relational model, structure mapping rules, and process algebraare represented as follows.

Table 2.2 Basic Model Structure of Decision Making of the Processing Chain

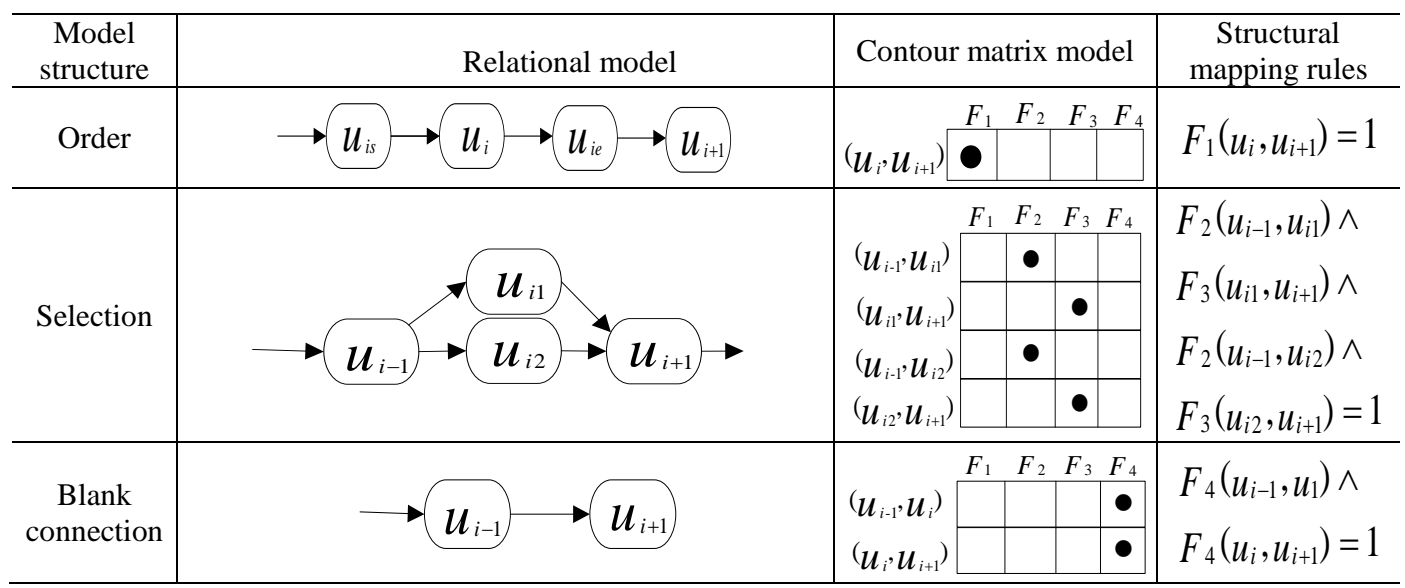

The accuracy constraint model is established in this paper to judge the relationship of edges in matrix $\mathrm{C}$. 
Table 2.3 Contour Accuracy Constraint Matrix

\begin{tabular}{|c|c|c|c|c|c|c|c|c|c|c|c|c|c|}
\hline & $F_{1}$ & $F_{2}$ & $F_{3}$ & $F_{4}$ & $F_{5}$ & $F_{6}$ & $F_{7}$ & $F_{8}$ & $F_{9}$ & $F_{10}$ & $F_{11}$ & $F_{12}$ & $F_{13}$ \\
\hline$u_{1}$ & 0 & & & & & 0 & 0 & 0 & & & & & \\
\hline$u_{2}$ & & 0 & & 0 & & & & & 0 & & & & \\
\hline$u_{3}$ & & & 0 & & 0 & & & & & 0 & 0 & & \\
\hline$u_{4}$ & & & & & & & & & & & & 0 & \\
\hline$u_{5}$ & & & & & & & & & & & & & 0 \\
\hline
\end{tabular}

In the table above, $\mathrm{F} 1$ to $\mathrm{F} 13$ respectively indicates the process means of rough turning, semi-refined turning, fine turning, bearizing, reaming, drilling, milling, boring, semi-fine boring, fine boring, grinding, accurate grinding, and abrading. Precision grades are represented by $\mathrm{u} 1$ to $\mathrm{u} 5, \mathrm{u} 1$ indicates IT13-IT11, which includes rough turning, rough boring, rough milling, rough planning, and drilling; u2 indicates IT10-IT9, which includes semi-refined turning, semi-fine boring, semi-fine milling, semi-fine planning, and bearizing; u3 indicates IT8 - IT7, which includes fine turning, fine boring, precision milling, fine planning, coarse grinding, and reaming.

u4 indicates IT7-IT6, which includes fine grinding and fine reaming; u5 indicates IT5-IT2, which includes abrading, super finishing, polishing, and honing.

Assuming that $[A \times F]$ is indicated by array $a[][]$, the precision constraint matrix is expressed with array $b[i][c[x]] ; \mathrm{t}$ indicates the number of rows of the array, uij indicates the processing method of $u_{i}$, and $m\left(u_{i}\right)$ indicates the number of processing methods under i accuracy grade. Progressive search of $a[][]$ is conducted, the elements that are not equal to zero are checked, and the column heading of this element is stored in array $c[x]$; $\mathrm{n}$ indicates the number of elements of array $c[x]$. The determination algorithm of the feature machining step chain is shown in Figure2-1. 


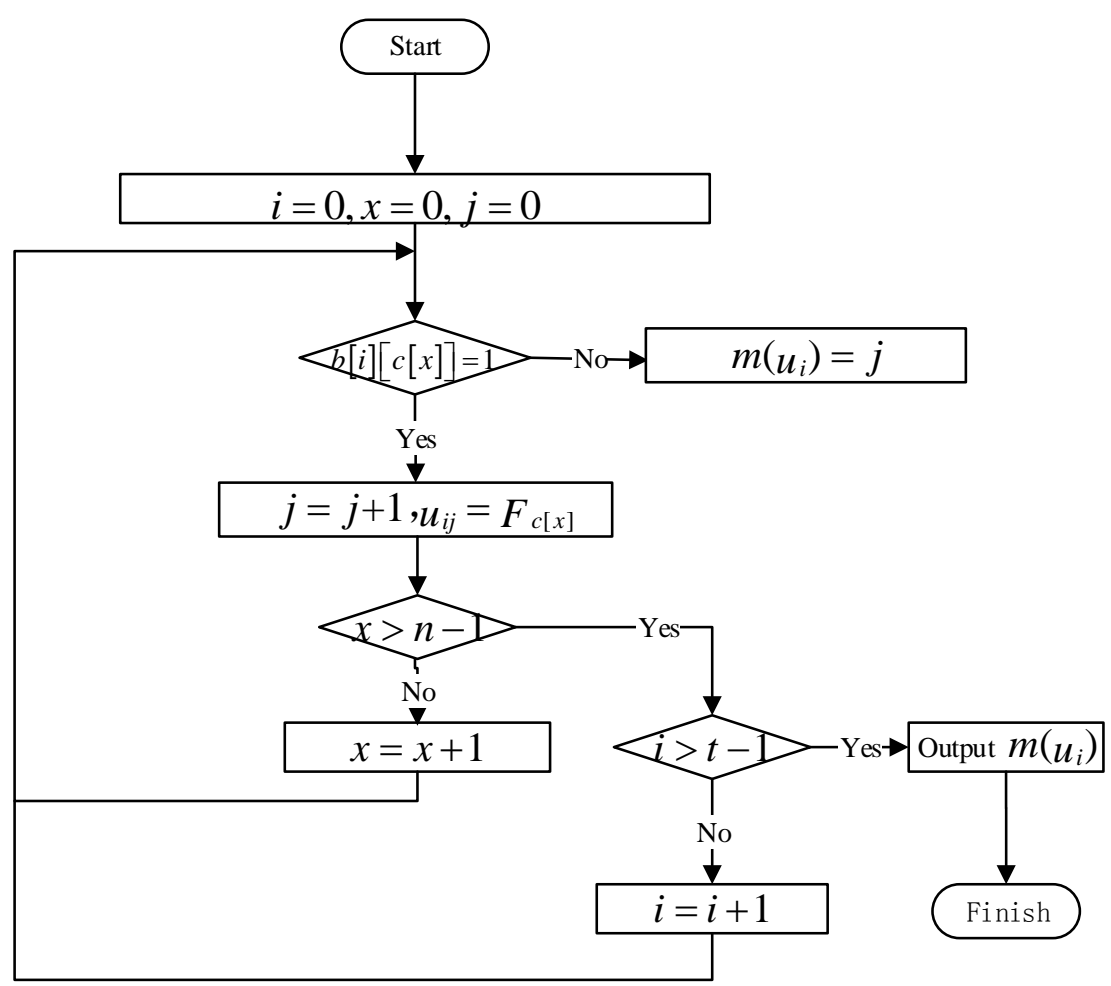

Figure 2.1 Determination Algorithm of the Feature Machining Step Chain

If $m\left(u_{i}\right)>1$, the edge relationship between $u_{i-1}$ and $u_{i}$ is "or divide," and the edge relationship between $u_{i}$ and $u_{i+1}$ is "or close."

If $m\left(u_{i}\right)=1$, the edge relationship among $u_{i-1}, u_{i}, u_{i}$ and $u_{i+1}$ is "sequential connection." If $m\left(u_{i}\right)=0$, the edge relationship between $u_{i-1}, u_{i}, u_{i}$ and $u_{i+1}$ is "blank connection"

After obtaining the above-described algorithm, the model of the relationship rules between edges can be obtained.

Table 2.4 Model of the Relationship and Rules between Edges

\begin{tabular}{|c|c|c|c|c|}
\hline & $F_{1}(C)$ & $F_{2}(C)$ & $F_{3}(C)$ & $F_{4}(C)$ \\
\hline$\left(u_{s}, u_{11}\right)$ & & $\bullet$ & & \\
\hline$\left(u_{11}, u_{2}\right)$ & & & $\bullet$ & \\
\hline$\left(u_{s}, u_{12}\right)$ & & $\bullet$ & & \\
\hline$\left(u_{12}, u_{2}\right)$ & & & & \\
\hline$\left(u_{2}, u_{3}\right)$ & $\bullet$ & & & $\bullet$ \\
\hline$\left(u_{3}, u_{4}\right)$ & & & & $\bullet$ \\
\hline$\left(u_{4}, u_{5}\right)$ & & & & \\
\hline$\left(u_{5}, u_{e}\right)$ & $\bullet$ & & & \\
\hline
\end{tabular}

By determining the type of edges between any two nodes, their logical relationship can be deduced. In the process of path planning, if a precision node and subsequent multiple process nodes have a logical relationship, then one of the process nodes is selected as the intermediate routing node. At the same time, the same numbers of path are replicated according to the number of subsequent nodes. If a blank connection exists, only one path 
exists between two nodes for sequential logic [12]. The process is then taken to the next accuracy node and continued. $S T$ indicates the processing step chain set of feature ${ }^{a_{i}}$ as follows:

$$
S T_{a_{i}}=\left\{S T_{i 1}, S T_{i 2} \cdots S T_{i p} \cdots S T_{i m}\right\} \text { and } S T_{i p}=\left\{s t_{i 1}, s t_{i 2}, \cdots s t_{i k}\right\}
$$

Hence, the process chain for each process feature obtained is from coarse to fine. Follow-up step sequencing is thus facilitated.

\subsection{Step Sequencing Model of the Process Route}

Process constraints are based on different machining phases and characteristics during the machining process. The constraint relationship between the characteristics during different processing phases is different. The working procedure cannot be classified before the process resource is selected. In mechanical processing, coarse and fine processing are certainly different working procedures. Hence, in this study, the machining process was divided into three stages, namely, rough machining, finishing, and semifinishing. The process feature sequencing decision is made in each phase.

First, scheduling of the parts main characteristics was implemented. Judgment of the matrix includes plane and hole constraint, surface of the primary and secondary level constraints, failure surface and non-failure surface constraints, dependent constraints, and similar constraints. Second, heat treatment process and auxiliary process scheduling are implemented.

Constraints can be divided into independent and related constraints. Independent constraints refer to constraints that are not between two features. Independent constraints include main and auxiliary features constraint. The purpose is to distinguish the main processing surface and its auxiliary features. The backbone is ensured, and the auxiliary process of characteristics is inserted into the process route. Related constraints can be divided into the following aspects.

Plane and hole constraint: It mainly refers to the processing of housing and bracket parts. Generally, such a class of parts can be both flat and have a hole or holes. The plane, which is usually an assembled benchmark, should be processed. The hole or holes are then processed based on the datum plane.

Benchmark relationships: Benchmark refers to the dots, lines, and planes that determine the other points, lines, and planes of the parts. Benchmark relationship denotes the relationship between features that are constituted when one or more characteristics become a benchmark for the mechanical processing of other characteristics.

Positioning relationship: The topological relationship between the characteristics that comprise the parts is not strictly required during process reasoning. However, at the time of processing, a positioning relationship may exist between two seemingly unrelated characteristics, that is, a feature's location size is determined by another characteristic.

Derivative relations: This relation implies a successively generated relationship between features. Before a certain feature is processed, another characteristic does not exist or cannot be processed. Such a relation is defined as derivative relation in this paper.

Accuracy requirement: In accordance with the parts function, the location accuracy requirement of characteristics, which is one of the most common characteristic of the relationship, is proposed.

Similar relationship characteristics: To ensure parts processing precision and achieve the lowest processing cost, mechanical processing organization is usually based on group technology. Hence, similar characteristics in the processing of parts may together constitute a relationship [13]. 
2.3.1 Process Characteristics Sequencing in Different Processing Phases: According to the primary and secondary side constraints, the characteristics to be processed are determined through backbone technology. Primary and secondary surface constraint matrices are shown in Table 2-5.

Table 2.5 Primary and Secondary Surface Constraint Matrices

\begin{tabular}{|c|c|c|c|c|c|c|c|c|c|c|}
\hline & $a_{1}$ & $a_{2}$ & $a_{3}$ & $a_{4}$ & $a_{5}$ & $a_{6}$ & $a_{7}$ & $a_{8}$ & $a_{9}$ & $a_{10}$ \\
\hline$b_{1}$ & $\bullet$ & & $\bullet$ & $\bullet$ & $\bullet$ & & & $\bullet$ & & $\bullet$ \\
\hline$b_{2}$ & & $\bullet$ & & & & $\bullet$ & $\bullet$ & & $\bullet$ & \\
\hline
\end{tabular}

In Table 2.5, $a_{i}$ refers to process characteristics, $b_{1}$ is the primary surface, and $b_{2}$ represents the secondary surface.

Technological characteristics-related constraints, however, are difficult to describe through the coloring methods for single technological characteristics.

The number of ordered planes to staining is established, and the order number for the mutual constraint relationship between two elements is described. This paper presents a color for the ordered number to describe the mutual constraint relationship between two elements.

$$
\begin{gathered}
B=\left\{a_{1}, a_{2}, \cdots a_{i}, \cdots a_{n}\right\} \\
U=\left\{\left(a_{1}, a_{2}\right), \cdots\left(a_{i}, a_{J}\right) \cdots\left(a_{n}, a_{n}\right)\right\} \\
F(\mathrm{x}, \mathrm{y})=\left\{F_{1}(x, y), F_{1}(x, y), \cdots F_{m}(x, y)\right\}
\end{gathered}
$$

In primary surface collection $\mathrm{B}$, the combination of two elements constitutes an ordered number called collection U. Each element in collection U represents a point on the plane, which correspondingly refers to the successive processing order of relevant process characteristics. Unified color set $\mathrm{F}(\mathrm{x}, \mathrm{y})$ represents plane and hole, benchmark, positioning, lineage, position accuracy, and similarity relationships.

$$
\begin{aligned}
& F_{1}\left(a_{i}, a_{j}\right)=1 \text { means hole ai attached to surface aj. } \\
& F_{2}\left(a_{i}, a_{j}\right)=1 \text { refers to process characteristic ai based on process characteristics aj. }
\end{aligned}
$$

$F_{3}\left(a_{i}, a_{j}\right)=1$ implies location dimension of ai depending on the location dimension of aj.

$$
F_{4}\left(a_{i}, a_{j}\right)=1 \text { means ai derived from aj. }
$$

$F_{5}\left(a_{i}, a_{j}\right)=1$ indicates that the position precision requirement of aj is higher than that of ai.

$F_{6}\left(a_{i}, a_{j}\right)=1$ means aj and ai belong to the similar feature (such a relationship will be disorderly).aj and ai are combined so that they can be processed in the same working procedure.

After identifying the above colors, the system automatically ranks the process feature, that is, in the Cartesian product of collection $\mathrm{A},{ }^{e_{i j}}=1$. Assuming that the matrix D value 
is stored in array $e[][]$, the color value of the order number is stored in 3D array $r[][][]$. The assignment algorithm is as follows:

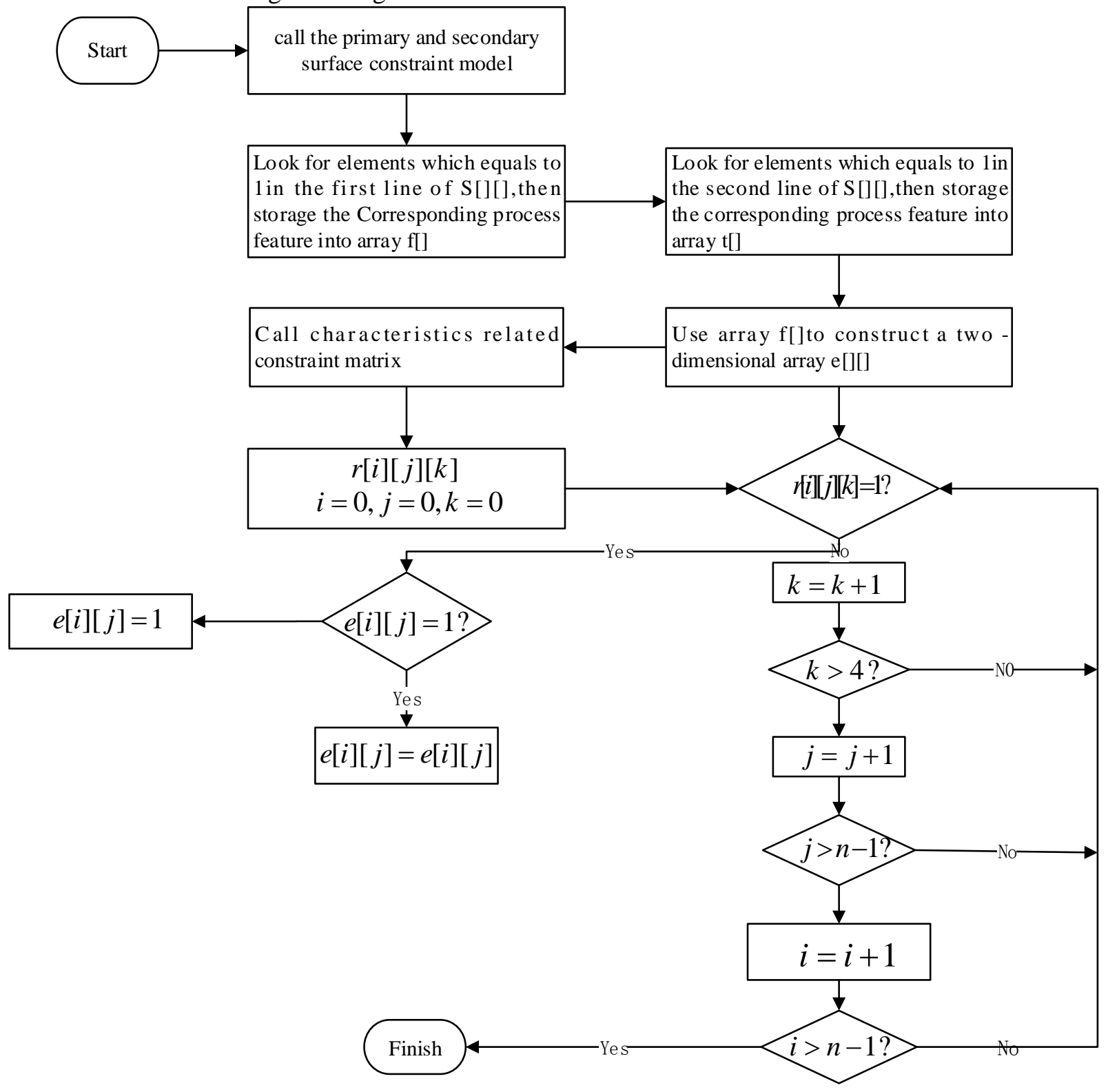

Figure 2-2. Assignment Algorithm

Based on the coloring situation of the order number, the system assigns a value to the Cartesian product matrix of technological characteristics according to the above algorithm. The matrix is shown below.

$$
D=[A \times A]=\left[\begin{array}{cccc}
a_{1} & a_{j} & & a_{n} \\
& {\left[\begin{array}{ccccc}
e_{11} & \cdots & e_{1 j} & \cdots & e_{1 n} \\
\vdots & & \vdots & & \vdots \\
e_{i 1} & \cdots & e_{i j} & \cdots & e_{i n} \\
\vdots & & \vdots & & \vdots \\
& & & \\
e_{n 1} & \cdots & e_{n j} & \cdots & e_{n n}
\end{array}\right] \begin{array}{c}
a_{1} \\
\vdots \\
a_{i} \\
a_{n}
\end{array}}
\end{array}\right.
$$


eij $=1$ means aj is located before ai. Every line of matrix D is searched. Sorting is implemented according to the number of 1 in each line, and the processing order of technological characteristics is outputted.

\subsubsection{Insertion of the Secondary Surface, Heat Treatment Process, and Auxiliary}

Process: The content above reveals how the process step chain set of each characteristic is obtained and how to obtain the processing order of technological characteristics. According to the processing sequence of technological characteristics, selecting different working step chains can form different process routes. However, the process route cannot be regarded as the final output process route. Heat treatment process and auxiliary process are required for the final output because the heat treatment process has a significant correlation with the green process and auxiliary work steps, such as inspection in the middle of the process, cleaning, burring, reasonable oiling, preventing parts from being knocked and wounded, and ensuring cleanliness; such steps are indispensable.

The heat treatment procedure follows the following principles.

Generally, processes such as normalizing, annealing, and tempering should be completed before machining to improve the machinability of metal.

To eliminate internal stress, aging treatment and conditioning are carried out before finishing but after rough machining.

Carburizing, quenching, tempering, and so on are often arranged after mechanical processing to improve the effect of the mechanical properties of parts.

If parts exhibit large deformation after heat treatment, the final machining process should also be arranged.

The arrangement of the secondary processing is as follows: processing before final finishing but after the main surface has reached a certain precision.

The arrangement of the assistive process is as follows: in the secondary process, inspection is the most important step. In addition to the self-check in each process, an inspection process should also be arranged at the end of the tough process stage, around the key working procedure and cycling between production workshops and after all parts have been finished.

A given process route has a unique work step sequencing, that is, no choice relationship exists between two work steps; they have an order connection relationship. The increase order node model is provided below.

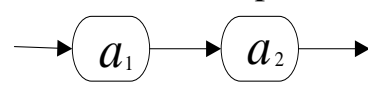

(a)Order connection (2 nodes

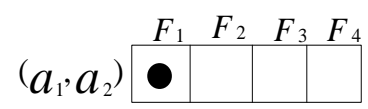

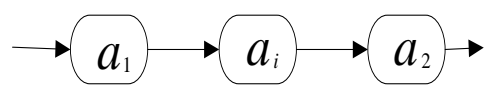

(b)Order connection (3 nodes)

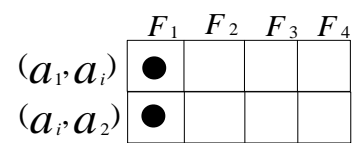

(c) Order connection PS model (2 nodes) $(d)$ Order connection PS model (3 nodes)

Figures (a) and (b) represent order connection structures made up of two and three nodes, respectively. (c) and (d) show the corresponding PS contour matrix. The Boolean expressions are

$$
F_{1}\left(a_{1}, a_{2}\right)=1_{\text {and }} F_{1}\left(a_{1}, a_{i}\right) \wedge F_{1}\left(a_{i}, a_{2}\right)=1 .
$$

\section{Process Resource Allocation based on Polychromatic Sets Theory}

Different work steps correspond to different process methods, and the choice of process method determines the choice of technological resources. After determining the 
sequence of working steps, process resource configuration should be carried out according to the personality of parts.

Unified color exists only when one or a few elements ai $\square \square \mathrm{A}$ exist in set A. The set of these elements ai $\square \square \mathrm{A}$ is called the first $\mathrm{k}$ unified colored individual expressed as

$$
A_{k}\left(F_{j}\right)=\left\{e, t, \cdots a_{i_{s}}, \cdots a_{i_{t}}\right\}
$$

All unified colored individuals $\mathrm{Fj}$ are expressed in the form shown below.

$$
A\left(F_{j}\right)=\bigcup_{k=1}^{m_{j}} A_{k}\left(F_{j}\right)=\left\{A_{1}\left(F_{j}\right), A_{2}\left(F_{j}\right), \cdots A_{k}\left(F_{j}\right) \cdots A_{m_{j}}\left(F_{j}\right)\right.
$$

Tectonic process resource collection and the Boolean matrix of unified colored individuals can be realized according to the process resource and the function they have.

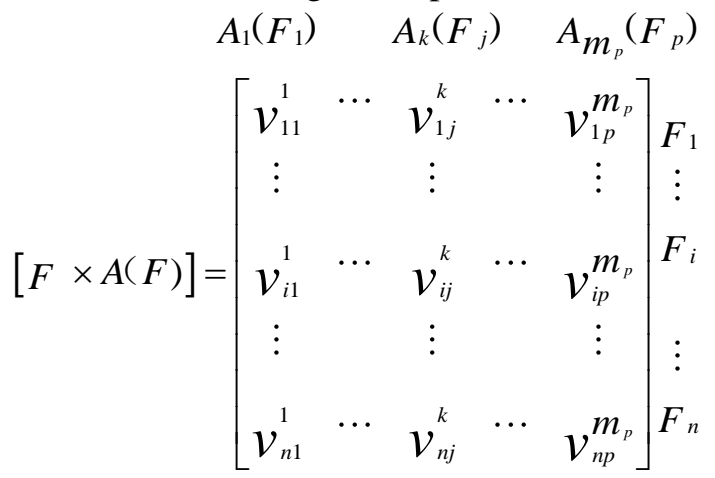

In the Boolean matrix, if $F_{i} \in A_{k}\left(F_{j}\right)$,then $v_{i j}^{k}=1$, otherwise, $v^{k}=1$.

When optimizing the allocation of resources of the process route, the resources (machine tool, cutting tool, cutting fluid, accessories) required in each work step are interact. To guarantee the independence of the request individual, we regard $\mathrm{Ak}(\mathrm{Fj})$ as the uniform colored individual. Se refers to the machine code, St represents the cutting tool code, Sl indicates the cutting fluid code, and Sm denotes the auxiliary material code.

Technological process $\mathrm{Fj}$ can be realized by a variety of resource combinations. When allocating resources, multiple work steps that can be aggregated into a process should be considered. Hence, the principles of resource selection and process description are mutually inverse.

(1) In each processing stage, to make the process as short as possible, adjacent work steps are recommended to use the same type of processing equipment as much as possible.

(2) On the basis of the first principle, when processing by using the numerical control machine tool between continuous steps, the same tools should be selected together as much as possible to shorten the tool change time.

In the process of allocating resources, when considering the two principles above to minimize the number of processes and number of tool change times, the following judgment model must be selected and utilized. 


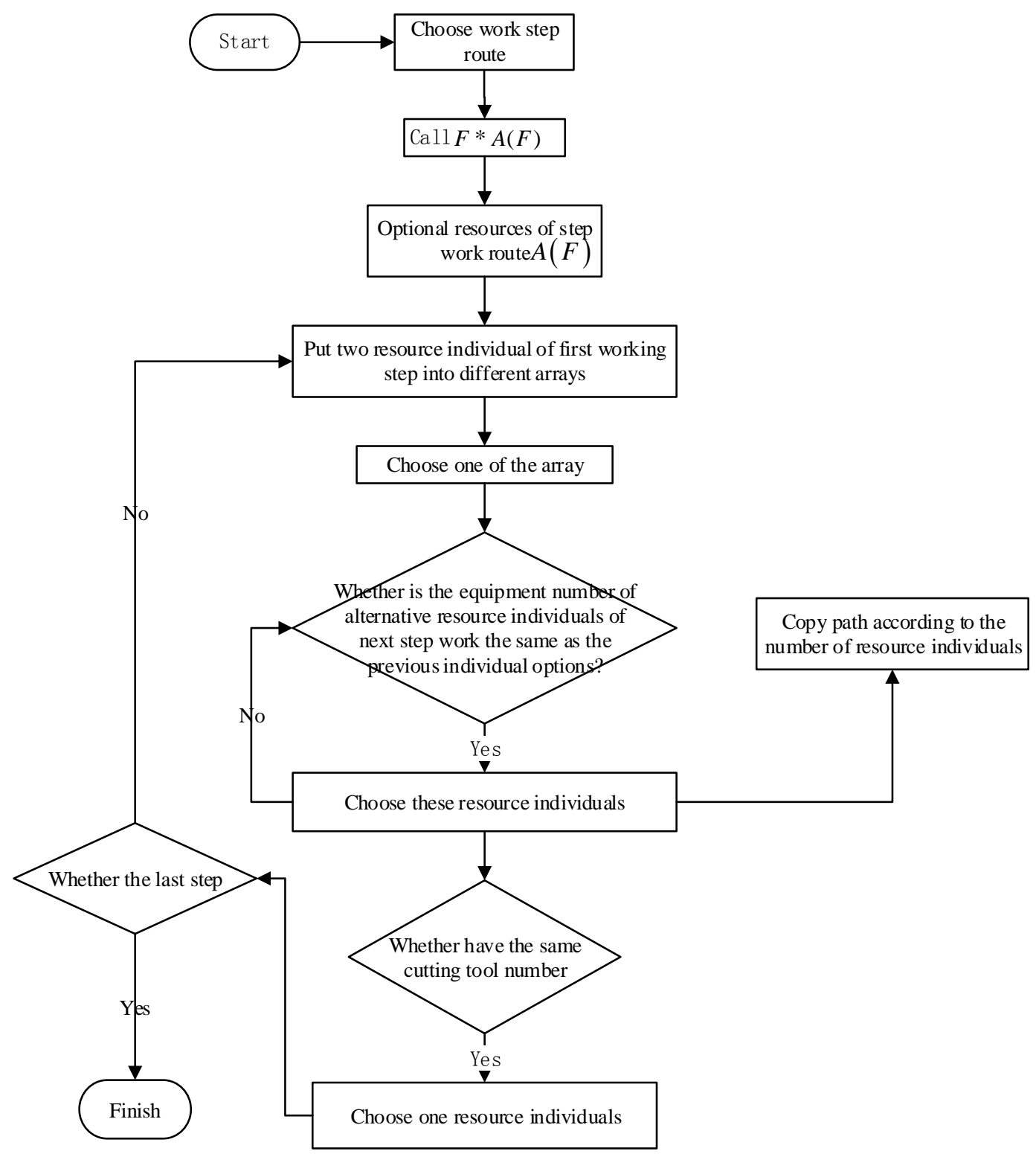

Figure 3-1. Process Resource Allocation Model

\section{Sample Verification}

We regarded the output shaft shown in Table 4.1 as an example to explain the planning process based on polychromatic sets. The material of the shaft is 45 steel. Through an analysis of the shaft, we found that its machining order is as follows: plane, outer circle, key slot, and chamfer. The process route is as follows: heat treatment, rough turn plane 2, centering, coarse turn outer circles, rough turn plane 1, centering, semi-finished turn outer circles, fine turn outer circles, end surface grind planes, and mill key slots. The shaft has a total of 17 characteristics that can be classified into five types; a unique number is provided to them from left to right in turn. Their accuracy requirements are shown in the table below. 


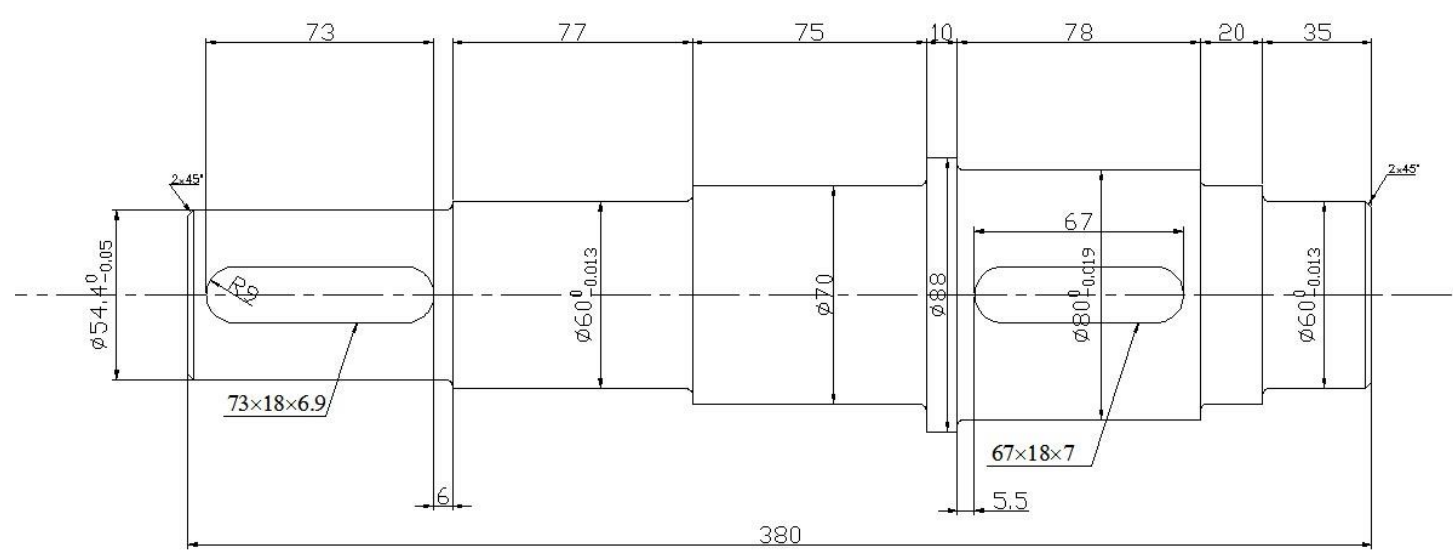

Table 4.1 Introduction of the Parts

\begin{tabular}{|c|c|c|}
\hline $\begin{array}{l}\text { Serial number of } \\
\text { characteristics }\end{array}$ & Name of characteristics & Precision requirement \\
\hline$a_{1}$ & center hole 1 & IT12 \\
\hline$a_{2}$ & plane1 & Ra12.5 \\
\hline$a_{3}$ & left side chamfer $1 * 2$ & IT12 \\
\hline$a_{4}$ & right side chamfer $2 * 2$ & IT12 \\
\hline$a_{5}$ & $\Phi 54.4 * 85$ & IT18(1.6) \\
\hline$a_{6}$ & Key slot 1 & IT9 \\
\hline$a_{7}$ & $\Phi 60 * 77$ & IT15(1.6) \\
\hline$a_{8}$ & $\Phi 70 * 75$ & IT11 \\
\hline$a_{9}$ & $\Phi 88 * 10$ & IT11 \\
\hline$a_{10}$ & $\Phi 80 * 78$ & IT6(1.6) \\
\hline$a_{11}$ & Key slot 2 & IT9 \\
\hline$a_{12}$ & $\Phi 70 * 20$ & IT11 \\
\hline$a_{13}$ & $\Phi 60 * 35$ & IT5(1.6) \\
\hline$a_{14}$ & Plane 2 & Ra12.5 \\
\hline$a_{15}$ & center hole 2 & IT12 \\
\hline
\end{tabular}

(1) Tectonic characteristics make up the working step matrix, as shown in Figure 2.1.

(2) The working step chain of a part's features is generated.

(3) The structure precision constraint matrix of the machining method is shown in Table 2.3. According to the algorithm shown in Figure 2-1, the working step chain of characteristics is determined. Given the limited space, this paper only shows the working step chain collection of the hole.

$$
\mathrm{S} T_{a_{i}}=\left\{S T_{i 1}, S T_{i 2}, S T_{i 3}\right\}=\left\{\left\{F_{4}, F_{2}, F_{3}, F_{8}\right\},\left\{F_{5}, F_{6}, F_{7}\right\},\left\{F_{5}, F_{6}, F_{8}\right\}\right\}
$$

(4) The process features are ordered in different stages. The rough machining stage is regarded 
(5) an example. According to the primary and secondary surface independent constraint matrices, the main processing surfaces are determined.

Table 4. 2 Primary and secondary surface independent constraint matrices

\begin{tabular}{|c|c|c|c|c|c|c|c|c|c|c|c|c|c|c|c|}
\hline & $a_{1}$ & $a_{2}$ & $a_{3}$ & $a_{4}$ & $a_{5}$ & $a_{6}$ & $a_{7}$ & $a_{8}$ & $a_{9}$ & $a_{10}$ & $a_{11}$ & $a_{12}$ & $a_{13}$ & $a_{14}$ & $a_{15}$ \\
\hline$b_{1}$ & $\bullet$ & $\bullet$ & & & $\bullet$ & & $\bullet$ & $\bullet$ & $\bullet$ & $\bullet$ & & $\bullet$ & $\bullet$ & $\bullet$ & $\bullet$ \\
\hline$b_{2}$ & & & $\bullet$ & $\bullet$ & & $\bullet$ & & & & & $\bullet$ & & & & \\
\hline
\end{tabular}

$B=\left\{a_{1}, a_{2}, a_{5}, a_{7}, a_{8}, a_{9}, a_{10}, a_{12}, a_{13}, a_{14}, a_{15}\right\}$

The contour constraint matrix between primary features is shown in Table 4.3.

Table 4.3 Contour Constraint Matrix between Primary Features

\begin{tabular}{|c|c|c|c|c|c|c|c|c|c|c|c|c|c|c|c|c|c|c|c|c|c|c|c|}
\hline & $\begin{array}{c}\hat{D} \\
\mathrm{E}\end{array}$ & 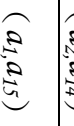 & 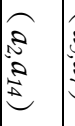 & 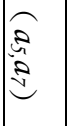 & 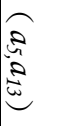 & 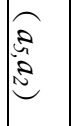 & 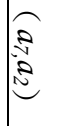 & 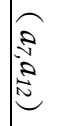 & $\begin{array}{c}2 \\
2 \\
0 \\
0 \\
0 \\
0\end{array}$ & $\begin{array}{l}2 \\
0 \\
\infty \\
0 \\
0 \\
0\end{array}$ & $\begin{array}{c}2 \\
\infty \\
\infty \\
0 \\
0 \\
0\end{array}$ & 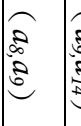 & $\begin{array}{l}8 \\
8 \\
0 \\
0\end{array}$ & $\begin{array}{c}8 \\
0 \\
0 \\
0 \\
0\end{array}$ & 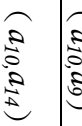 & है & 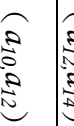 & 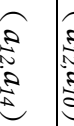 & 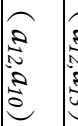 & 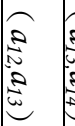 & $\begin{array}{l}2 \\
0 \\
c \\
0 \\
0 \\
0\end{array}$ & 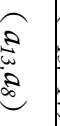 & $\begin{array}{l}8 \\
0 \\
E \\
E\end{array}$ \\
\hline$F_{1}$ & O & & & & & & & & & & & & & & & & & & & & & & 0 \\
\hline$F_{2}$ & 0 & & & & & 0 & 0 & & & 0 & & & 0 & & 0 & & & 0 & & & 0 & & \\
\hline$F_{3}$ & & & & & & & & & & & & & & & & & & & & & & & \\
\hline$F_{4}$ & & & & 0 & 0 & & & 0 & & & 0 & & & & & 0 & & & 0 & & & 0 & \\
\hline$F_{5}$ & & & & & & & & & & & & & & & & & & & & & & & \\
\hline$F_{6}$ & & 0 & 0 & & & & & & 0 & & & 0 & & 0 & & & - & & & 0 & & & \\
\hline
\end{tabular}

According to the algorithm in Figure 2-2, the order of process features can be obtained at all stages of processing.

Rough achining

stage:

$a_{14} \rightarrow a_{15} \rightarrow a_{9} \rightarrow a_{10} \rightarrow a_{12} \rightarrow a_{13} \rightarrow a_{2} \rightarrow a_{1} \rightarrow a_{8} \rightarrow a_{7} \rightarrow a_{5}$

Semi finishing stage: $a_{10} \rightarrow a_{13} \rightarrow a_{7} \rightarrow a_{5}$

Finishing stage: $a_{10} \rightarrow a_{13} \rightarrow a_{7} \rightarrow\left(a_{1} \rightarrow a_{15}\right)$

Ultra precision machining stage: $a_{13} \rightarrow a_{7}$

Secondary surface processing: $a_{6} \leftrightarrow a_{11} \leftrightarrow a_{3} \leftrightarrow a_{4}$

(6) The heat treatment process is inserted. Part drawing requires conditioning treatment, so

the

(7) Heat treatment should be inserted before processing. The result based on the machining method can be expressed as follows:

$$
\begin{aligned}
& F_{0} \rightarrow F_{1} \rightarrow F_{6} \rightarrow F_{1} \rightarrow F_{1} \rightarrow F_{1} \rightarrow F_{1} \rightarrow F_{1} \rightarrow F_{6} \rightarrow F_{1} \rightarrow F_{1} \rightarrow F_{1} \\
& \rightarrow F_{2} \rightarrow F_{2} \rightarrow F_{2} \rightarrow F_{2} \rightarrow F_{2} \rightarrow F_{2} \rightarrow F_{2} \rightarrow F_{11} \rightarrow F_{11} \rightarrow F_{11} \rightarrow F_{11} \\
& \rightarrow F_{7} \rightarrow F_{7} \rightarrow F_{1} \rightarrow F_{1}
\end{aligned}
$$

(8) The process resource is allocated. The optional process resources involved in the process 
route are shown in Table 4.4.

Table 4.4 Details of the Parts

\begin{tabular}{|c|c|c|c|c|}
\hline $\begin{array}{l}\text { Process } \\
\text { means }\end{array}$ & Machine & Tool & $\begin{array}{l}\text { Cutting } \\
\text { fluid }\end{array}$ & $\begin{array}{l}\text { Supplementary } \\
\text { materials }\end{array}$ \\
\hline$F_{1}$ & CA6140/C5112/CK6132 & G8/K30/YW2/YG15 & $M B-132$ & - \\
\hline$F_{2}$ & CA6140/C5112/CK6132 & G6/YG6X/M10 & $M B-132$ & - \\
\hline$F_{3}$ & CA6140/C5112/CK6132 & $Y G 3 X$ & $M B-132$ & \\
\hline$F_{6}$ & Z5150/XCJ50 & $\begin{array}{c}\text { Straight shank drill M4 } \\
\text { /Reamer M4 }\end{array}$ & $40 \mathrm{Cr}$ & $\begin{array}{l}\text { Drill chuck } \\
\text { /Drill bushing }\end{array}$ \\
\hline$F_{11}$ & $M 1432 C$ & $\begin{array}{c}\text { Tapered wheel/ } \\
\text { wheel500*50*305*10 }\end{array}$ & $M B-103$ & - \\
\hline$F_{7}$ & X6140/XK7132 & $\begin{array}{c}\text { Key slot milling cutter } \\
\text { M9/M16/20 }\end{array}$ & $M B-113$ & - \\
\hline
\end{tabular}

According to the craft route and the algorithm shown in Figure 2.3, the system eventually assembles the steps as a process.

$$
\begin{aligned}
& F_{0} \rightarrow F_{1} \rightarrow F_{6} \rightarrow\left(F_{1} \rightarrow F_{1} \rightarrow F_{1} \rightarrow F_{1}\right) \rightarrow F_{1} \rightarrow F_{6} \rightarrow\left(F_{1} \rightarrow F_{1} \rightarrow F_{1}\right) \\
& \rightarrow\left(F_{2} \rightarrow F_{2} \rightarrow F_{2} \rightarrow F_{2}\right) \rightarrow\left(F_{3} \rightarrow F_{3} \rightarrow F_{3}\right) \rightarrow\left(F_{11} \rightarrow F_{11}\right) \rightarrow\left(F_{11} \rightarrow F_{11}\right) \\
& \rightarrow\left(F_{7} \rightarrow F_{7}\right) \rightarrow\left(F_{1} \rightarrow F_{1}\right)
\end{aligned}
$$

The final resource allocation scheme is shown in italics in Table 4.2 and expressed in polychromatic sets as follows:

$$
\begin{aligned}
& A_{1}\left(F_{0}\right) \vee A_{1}\left(F_{1}\right) \vee A_{1}\left(F_{6}\right) \vee A_{2}\left(F_{1}\right) \vee A_{1}\left(F_{1}\right) \vee A_{1}\left(F_{6}\right) \vee A_{3}\left(F_{1}\right) \vee A_{3}\left(F_{2}\right) \\
& \vee A_{2}\left(F_{3}\right) \vee A_{4}\left(F_{11}\right) \vee A_{3}\left(F_{11}\right) \vee A_{1}\left(F_{7}\right) \vee A_{2}\left(F_{1}\right)
\end{aligned}
$$

\section{Conclusion}

The sorting problem in process planning and the allocation of resources based on polychromatic sets theory were studied. A technological path generation algorithm was proposed based on polychromatic sets theory. An organic integration of process planning and technology resource allocation by using polychromatic sets was presented. The proposed theory and method have the following advantages.

(1) The use of hierarchical process decision in stages meets customer requirements without losing generality.

(2) The knowledge expression and reasoning of each stage and expressing form were described by using polychromatic sets theory. Complex process decision making can be carried out with the unified mathematical model. Moreover, the combination of the process planning model and the resource configuration model at a logical level was presented. 


\section{Acknowledgment}

The research is supported by the National Natural Science Foundation of China (NSFC) under Grant No. 51205429 and the Technology Pillar Program during the 12th Five-year Plan Period of China under Grant No. 2012BAF01B01 .

\section{References}

[1] XIAO Wei-yue. Constraint matching algorithm for generating main sequences of process plan in CAPP [J].Journal Of Computer-aided Design \& Computer Graphics.2001, 13(1):40-43.

[2] ZHANG Xiao-dong,ZHANG Zhi-qiang,CHEN Jin.Constructing method of production decision making expert system based on interactive simulation[J].Computer Integrated Manufacturing Systems.2013(2),19(2):404-410

[3] Liu En-fu, Fang Yi-xiang, Liu Xiao-yang, Li Zong-bin. Study on the strategic decision of processing scheme for box-type parts based on polychromatic sets theory[J]. ICCET 2010 - 2010 International Conference on Computer Engineering and Technology, Proceedings, v5. 2010: V5161 V5164.

[4] HU Yu-jin, ZHANG Zheng-yi, Ling Ling et al. Process sequence optimization based on process constraint matrix. China Academic Journal, 2009, 20(9):1062-1066.

[5] HUANG Wei-jun, CAI Li-gang, HU Yu-jin et al. Process planning optimization based on genetic algorithm and topological sort algorithm for digraph [J] Computer Integrated Manufacturing Systems.2009, 15(9):1770-1778.

[6] OUYANG Hua-bing,SHEN Bin.STEP-NC oriented process planning optimization based on hybrid genetic algorithm[J].Computer Integrated Manufacturing Systems.2012,18(1):68-75.

[7] ZHANG Guan-wei, ZHAO Xiang-song, LI Jia et al. Method of operation optimization sequencing using genetic algorithm[J].Computer Integrated Manufacturing Systems.2005,11(2):242-246.

[8] Alluru Gopala Krishna·K, Mallikarjuna Rao. Optimization of operations sequence in CAPP using an ant colony algorithm [J].Int J Adv Manuf Technol.2006, 29:159-164

[9] LIU Xiao-jun,YI Hong,NI Zhong-hua.Application of ant colony optimization algorithm in process planning optimization[J].J Intell Manuf.2013,24:1-13.

[10] ZHOU Xian.A study of method for fixture design based on polychromatic sets theory[J]. Science Technology and Engineering. 2014,14(15):83-87

[11] DU Xuan, LI Zong-bin, ZHANG Guo-hui. Job shop printed circuit board assembly optimization based on model constrained by poly-chromatic sets [J].2009, 20(22):2687-2693.

[12] WANG Song, LI Yun-long, CHEN Fu-min. Process planning and resource allocation in aircraft connection assembly based on poly-chromatic sets [J].Computer Integrated Manufacturing Systems.2013, 19(2):411-420.

[13] JIANG Shao-fei, PAN Shuang-xia, FENG Pei-en et al. Description of feature combinations of part and its application for CAD/CAPP integration[J].Journal of computer-aided design \&computer graphics.2002,14(8),781-785

\section{Authors}

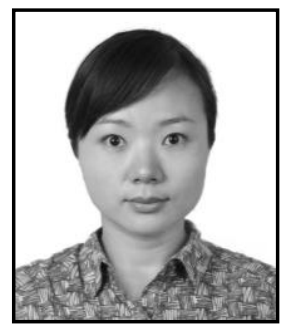

Lei Qi, She received her DR. degree in mechanical engineering from Chongqing university in Chongqing, China. She is currently an associated professor in Chongqing University. Her research interest is mainly in the area of Industry Engineering, Production Management and Manufacturing System Engineering. She has published several research papers in scholarly journals in the above research areas.

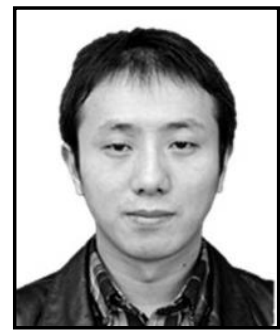

Li Xianwang, He received his DR. degree in mechanical engineering from Chongqing University in Chongqing, China. He is currently a lecturer in Guangxi University. His research interest is mainly in the area of Manufacturing Information and Networked Manufacturing. 


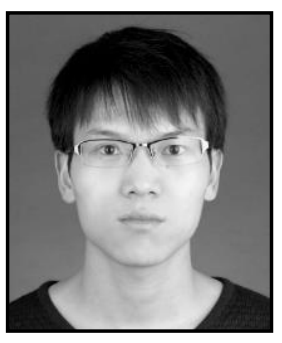

Guo Weifei, He is currently a graduate student who majors in industry engineering in College of Mechanical Engineering, Chongqing University in Chongqing, China. His research interest is mainly in the area of Manufacturing Information and Networked Manufacturing.

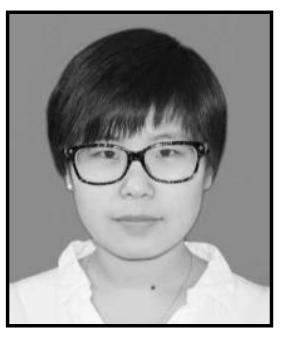

Li Xiaoxin, She received her M.S. degree in industry engineering from College of Mechanical Engineering, Chongqing University in Chongqing, China. Her research interest is mainly in the area of Manufacturing Information and Networked Manufacturing. 
International Journal of Grid and Distributed Computing Vol. 9, No. 9 (2016) 